\title{
Fractal Analysis of Morphological Parameters of the Structure Materials
}

\author{
Makarenko K.V., Nikitin A.A. \\ makkon1@yandex.ru| zzzalexzzz95@gmail.com \\ BSTU, Bryansk, Russia
}

\begin{abstract}
It is proposed to use the methods of fractal analysis to determine the morphological characteristics of the structure of structural materials. The questions of fractal aggregation of particles in the process of crystallization of ductile iron are considered, an austeniticgraphite cell is used as an elementary particle. Based on the mesh method, images of the microstructure of ductile irons are analysed and conclusions are drawn about the similarity of the nature of the process of their crystallization and fractal aggregation of particles. Based on the calculated fractal dimensions, a theory is proposed to explain the features of the crystallization process of ductile irons.
\end{abstract}

Keywords: fractal analysis, dimension, cluster, aggregation, material, cast iron, graphite, inclusions, crystallization.

\section{Introduction}

The fractal geometry $[3,8]$ allows to describe geometrical objects difficult in structure through one parameter which the fractal dimension (D) is. For the ideal sphere in 3-dimensional space of $\mathrm{D}=3$, a circle in plane $\mathrm{D}=2$, a circle and a straight line of $\mathrm{D}=1$. For the simplest geometrical figures the fractal dimension coincides on value with Euclidean and topological dimensions. Fractional dimensions, which for the inclusions studied in the plane of a shlif will be in an interval of $\mathrm{D}[3,8]$ are characteristic of objects, difficult in a form of an outer surface. For the description of difficult objects to which, in particular, borders of grains belong on surfaces of a shlif or a relief of a surface of a break it is possible to use multifractal methods of the description of their geometrical parameters [1,2].

For the description of the graphitized cast iron of the phenomena connected with natural arrangement of graphite inclusions in structure arising at crystallization in the real work methods of a fractal clustering (aggregation) of substance were used. It is necessary to clear up the terminology used at the description of processes of formation of fractal clusters. In material science the cluster theory are associated, first of all, with the structure of liquid.

In terms of the theory of fractal aggregation, the term "cluster" has broader value and is used for the description of various structures arising in the course of their interaction or aggregation [4]. The theory of fractal aggregation is applied to the description of various physical processes [13].

Originally the term "fractal cluster" was applied to the description of the aggregation connected with formation of branched structures. The first can consider model of Witten Sander [6] according to which the particles generated by the program gradually join the germ, which is originally entered into the limited plane. At touch they are attached to it, at the expense of it there is a growth of the branched aggregate (fig. 1, a). Such model received the name DLA (Diffusion Limited Aggregation) in the Russian transcription ODA (The Aggregation Limited to Diffusion). The aggregate arising in such conditions is fractal, i.e. has geometrical characteristics of self-similarity, and density of particles in it changes under the power-law in process of removal from the center.

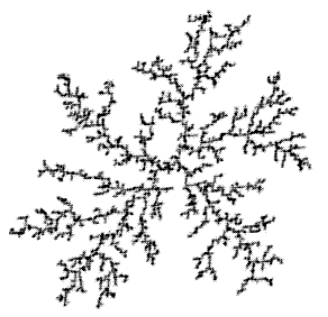

a)

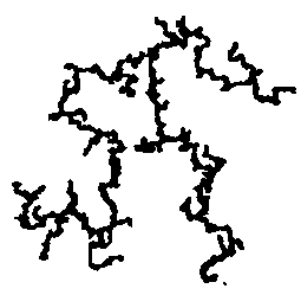

b)
Fig. 1. Kinds of fractal clusters: $a-$ the cluster corresponding to the DLA model; $b$ - the cluster formed on the CCA model [6]
Other model of aggregation of particles, which gained the greatest distribution - the CCA, model (Cluster - Cluster Aggregation). According to this model forming of the cluster aggregate is carried out by a join path of small clusters, which at the initial stage form according to the DLA model or its modifications. Such units have more friable building, than in the DLA model that affects reduction of their fractal dimensions (fig. $1, b)$.

The technique of studying of fractal clusters consists in determination of fractal dimension and the subsequent choice of the computer model, which is the most adequately describing structure formation conditions.

For determination of fractal dimensions use various methods: physical measurements, method of molecular adsorption, method of grids, correlation functions [10]. The geometrical difference of fractal structures from accidental distribution can be explained on the example of two images of particles in the square plane on a side of L (fig. 2).

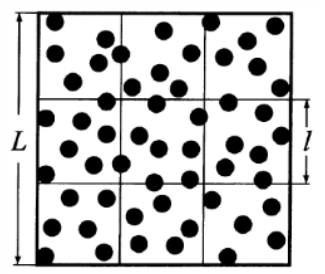

a)

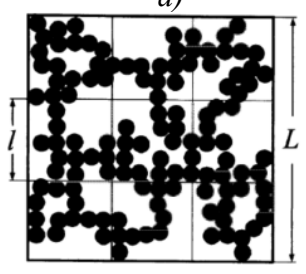

c)

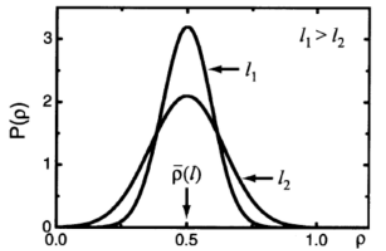

b)

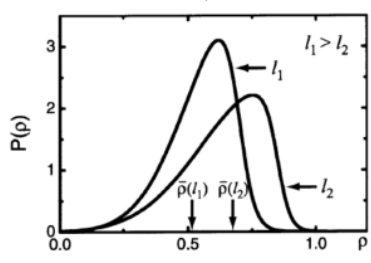

d)
Fig. 2. Systems of distribution of particles: a - the

deterministic distribution; $b, d$ - statistical functions of distribution of local density for the corresponding systems; $c$ - fractal distribution [10]

If to break a square into smaller cells with the party of $l$, then average mass density for number of cells of $i$ can be determined on a formula:

$$
\bar{\rho}_{i}(l)=\frac{\left[M_{i}(l)\right]_{i}}{l^{d}},
$$

where $M_{i}(l)$ - average particulate mass in cell $i$ with a size $l, d$ euclidean dimensionality. When determining statistical function of distribution density of particles value $\bar{p}_{l}(l)=$ const (fig. 2 , $b)$, and their mass is proportional to the cell size $\left[M_{i}(l)\right]_{i} \infty l^{d}$. Density can be determined as:

$$
\bar{\rho}=\frac{m}{\bar{a}^{d}},
$$

where $m$ - mass of one particle, $\bar{a}$ - average distance between particles. 
For the image submitted in figure $2, a$, the distance $\bar{a}$ characterizes the system of distribution of particles. On the contrary, in distribution of particles (fig. 2, c), characterized by their certain aggregation, density $\bar{p}_{l}(l)$ will not be a constant and changes from a cell to a cell. Most accurately, it can be tracked at change of size $l$ of a cell of a grid (fig. 2, d). In this case the mass of particles in a cell is proportional to its size $\left[M_{i}(l)\right]_{i} \infty l^{D_{f}}$ and depends on $D_{f}$ - fractal (fractional) dimensionality. Can determine average mass density for the system of cells (fig. 2, c) by a formula:

$$
\bar{\rho}_{i}(l)=\frac{\left[M_{i}(l)\right]_{i}}{l^{d}} \propto l^{D_{f}-d} .
$$

The image of structure proportional $l^{D_{f}}$ is considered fractal in statistical sense. This approach underlies a method of grids which is used in work for the fractal analysis of images of microstructures of graphitized cast irons.

Now methods of a fractal formalism are widely applied to the solution of various class of the tasks connected with the description of structure of the materials and processes proceeding in them $[5,9,11,12,14,15]$.

\section{Methodology for the of carrying out researcher}

As a result of a research of space distribution of graphite inclusions in high-strength cast iron, it there was suggestion, that at the heart of the mechanism of forming of chains of a graphite phase, the model a cluster - cluster aggregation lies (CCA) [7]. For the purpose of identification of the key geometrical parameters which are obtained in the course of crystallization of cast iron of the aggregation consisting of eutectic cells we will carry out the fractal analysis of images of a microstructure of high-strength cast iron.

For the description of the phenomena arising at crystallization of cast iron connected with natural arrangement of graphite inclusions in structure used methods of a fractal clustering (aggregation) of substance.

Cast iron for a research was melted in the induction furnace of industrial frequency IChT-1. Chemical composition of the melted cast iron, \% mass fraction:3,17 - 3,2 C; 2,9-3,1 Si; 0,6 - 0,7 Mn; 0,06 P; 0,03 S; 0,05-0,06 Mg.

Samples for a research of a microstructure filled in in dry sandy-argillaceous forms, they represented cylindrical harvesting $\varnothing 35 \times 300$. In addition, tidal samples and elements of gating casting system - profits and air gates have been exposed to microstructural studies.

Studying and registration of a microstructure were carried out on the analytical computerized complex which is created on the basis of the inverted metallographic microscope Leica DM IRM.

For identification of primary structure of cast iron of a microsection were exposed to thermal etching by a special reactant. For receiving a reactant of $5 \mathrm{~g}$ of picric acid $(\mathrm{C} 6 \mathrm{H} 2(\mathrm{NO} 2) \mathrm{OH})$ and $20 \mathrm{~g}$ of a caustic natr $(\mathrm{NaOH})$ dissolved in $100 \mathrm{ml}$ of the distilled water. Etching of microsection was carried out at a temperature of $70^{\circ} \mathrm{C}$ with endurance in a reactant of 40 min. After endurance at the set temperature of a microsection were cooled in a reactant to room temperatures. Microstructures cast iron after thermal etching are presented in fig. 3, $a$.

The cast iron microstructure fragment (fig. 3, a) after the corresponding processing was used for the subsequent fractal analysis of images. By preparation of a microstructure for the analysis made the following assumptions stated in the previous head. Graphite inclusions in fusion at eutectic crystallization arise gradually. It, in particular, is defined by the platform appearing on cooling curves at eutectic crystallization of cast irons. Though their growth at crystallization happens in different

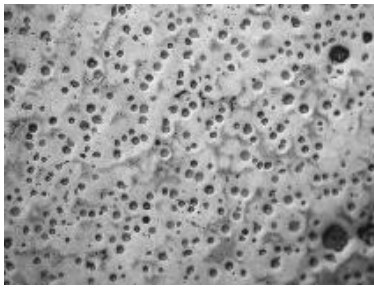

a)

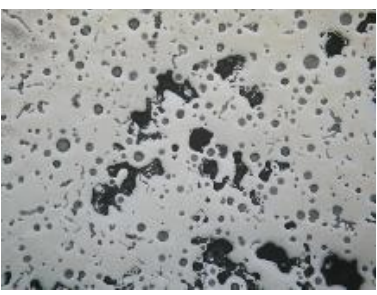

b)
Fig. 3. Microstructure in the studied samples

nodular cast iron: $a$-distribution of graphite inclusions

(thermal etching), $\times 80 ; b$-distribution of graphite

inclusions in a zone of shrinkable porosity, (microsection not etched), $\times 100$

periods of time, they usually have the similar sizes. Initial particles for formation in fusion of cast iron of cluster units are the eutectic cells consisting of graphite and an austenitic shell. Contact of these cells at crystallization of cast iron happens through austenitic shell as a result of which cells are welded to each other.

Studying distribution of graphite inclusions in areas of shrinkable porosity (fig. $3, \mathrm{~b}$ ), determined the average size of eutectic cells at which their contact is observed:

$$
D_{\text {eut.cells }} \approx 2,5 D_{g r} \text {, }
$$

where $D_{g r}$ - diameter of graphite inclusions.

By preparation of the image for the computer analysis (a fragment of fig. 3, a) settlement eutectic cells were the basis (fig. 4).

At distribution of particles in the plane of a microsection the contact of the circles characterizing spherical eutectic cells has to happen in one point, but on the submitted image some of them cross each other. It will be explained by the fact that in the

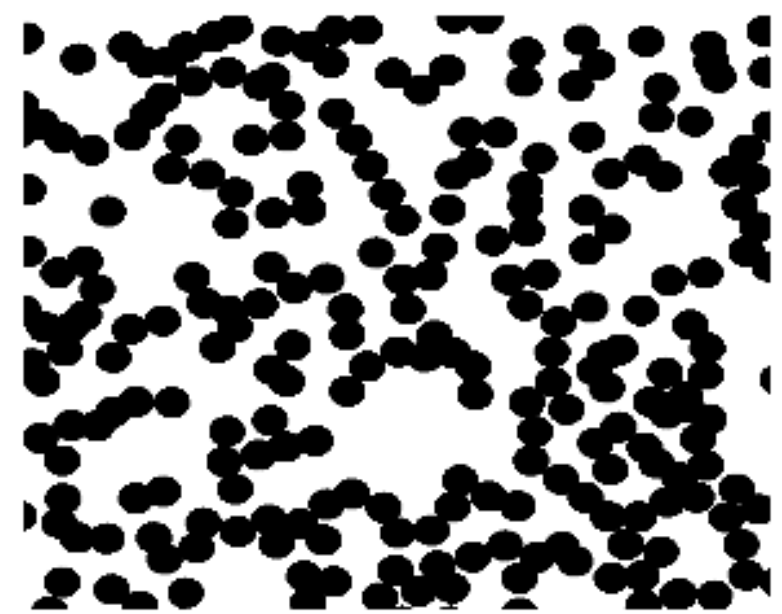

Fig. 4. The initial image for the computer analysis

analysis the eutectic cells located out of the plane of a microsection were considered. Use in the analyzed image of similar cells allows to consider the received structure, for the analysis of the image as spatial distribution of particles.

The fractal analysis of the received image was made with use of the ImageJ program and the additional FracLac module which works with binary (black-and-white) images and allows to make calculation of fractal dimensions in several ways. For the analysis of distribution of eutectic cells in structure of highstrength cast iron the algorithm of determination of fractal dimensions by a method of grids [16] at which the image of the unit breaks a grid into cells of given sizes was used (fig. 5, $a$ ). Scanning of the image of the unit is carried out for several cycles, at the same time on each subsequent cycle the sizes of cells of a grid increase (fig. $5, b$ ). The fractal dimension of the analyzed binary image of the aggregate is calculated on a formula: 


$$
D=\lim _{\varepsilon \rightarrow 0} \frac{\ln N_{\varepsilon}}{\ln \varepsilon}
$$

where $\varepsilon-$ grid cell size, $N_{\varepsilon}-$ quantity of cells of the size $\varepsilon$, containing pixels of the image of the aggregate. The size of a cell $(\varepsilon)$ is defined as the relation of the area of a cell to the total area of the image.

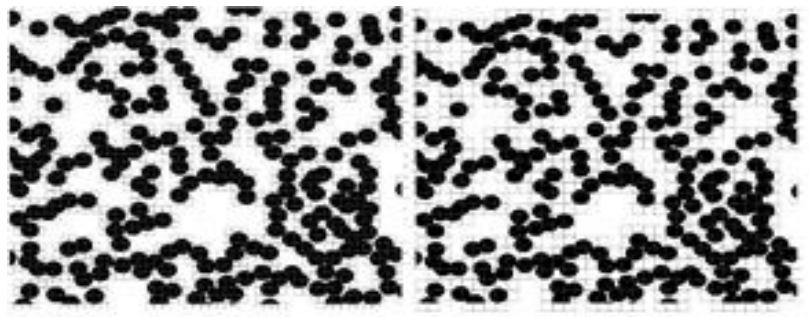

Fig.5. Results of individual scan cycles the studied unit is grid with cells of various dimensions: $a, b$ - grid size of cells 6 and 10 pixels respectively

Geometrically probabilistic fractal dimension of $\mathrm{D} \beta$ in the program module FracLac is determined by an inclination of the regression line calculated by a method of the least squares in coordinate $(-\ln \varepsilon)-\ln N_{\varepsilon}$. For obtaining average value of fractal dimension of the image the reference point location for scanning of the image of the unit a grid changes several times. Thus, primary scanning is the general for all image, and the subsequent with the changed reference points - local scanning of certain areas. After determination of fractal dimensions for various reference points the average value which is fractal dimension of the image is calculated. The calculated fractal dimension allows to choose model in compliance to which under the set conditions there is an aggregation of eutectic cells.

\section{Results of researches}

In fig. 6, $a$ the graphic dependence $(-\ln \varepsilon)-\ln N_{\varepsilon}$, received on the basis of design data of the fractal analysis of the image of the aggregate of distribution of eutectic cells in structure of highstrength cast iron is presented. The built line of regression corresponds to linear model:

$$
\ln N_{\varepsilon}=-1,79 \ln \varepsilon+0,33 \text {. }
$$

The coefficient of determination of $r^{2}$ is equal to 0,99 .

The coefficient at a predictor $(-\ln \varepsilon)$ defines fractal dimension fora given position of a grid of the image $\left(D_{\beta}=1,79\right)$.

The fractal dimension of the image $D_{\beta}$, calculated as mean value for four provisions of grids is equal to 1,75 . This value of fractal dimension corresponds to CCA - model of the cluster which is formed at association of particles in three-dimensional space.

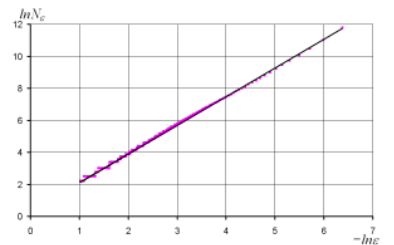

a)

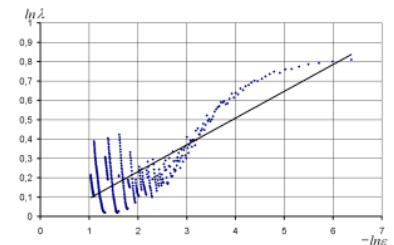

b)
Fig. 6. The graphic dependences received on the basis of design data with use of the module for the fractal analysis of FracLac 2.5: $a$-determination of fractal dimension of the image; $b$-determination of lacunarity of the fractal unit

Important parameter for the description of stochastic fractal structures is the lacunarity. The term "lacunarity" was for the first time used by B. Mandelbrot [1] for the description of unevenness of distribution of galaxies in an observed part of the Universe. Actually the lacunarity is a measure of heterogeneity of filling of space with an object. The lacunarity is higher, the more in the studied distribution is available empty areas. Similarly in the analyzed image of a microstructure of cast iron in the plane of a microsection a part of graphite inclusions is hidden by a metal matrix which forms perlitny areas, the program they are perceived as emptiness. Measure of lacunarity $(\Lambda)$ in the used program is change of density of the image of the aggregate when scanning by a grid of cells of various sizes. For its calculation the following formula is used:

$$
\Lambda=\left(\frac{\sigma}{\mu}\right)^{2}
$$

where $\sigma$ - standard deviation of weight (for the bitmap - the number of pixels) the fractal unit in cells of a grid of given size $\varepsilon, \mu$-average value of mass of the unit in cells of given size $\varepsilon$.

Similarly, as well as the fractal dimension, lacunarity is determined by an inclination of the regression line in coordinates $(-\ln \varepsilon)-\ln \Lambda$. For the fractal analysis of the image of distribution of eutectic cells to the planes of a microsection the received graphic dependence for determination of lacunarity is presented in fig. $6, \mathrm{~b}$. The regression equation for the line of a trend:

$$
\ln \Lambda=-0,14 \ln \varepsilon+0,04
$$

Lacunarity in the studied fractal aggregate $\Lambda=0,14\left(r^{2}=0,6\right)$, that indicates high uniformity of distribution of eutectic cells in volume of cast iron with spherical graphite. Despite rather low value of coefficient of determination $r^{2}$, the accurate tendency to positive correlation is traced.

Thus, the carried-out analysis of the image of eutectic cells in the plane of a microsection showed that the studied cluster unit differs in low lacunarity.

\section{Discussion of results}

The received results allow to develop process model crystallization of cast iron and to explain features of arrangement of graphite inclusions in its structure.

A certain fractal dimension $\left(D_{\beta}=1,75\right)$ corresponds a cluster - cluster model of aggregation (CCA) that is determined by the generalized databases of the existing models [6]. Such cluster has rather high density of distribution of graphite inclusions that is explained by rather low lacunarity.

According to chosen as model aggregation crystallization of high-strength cast iron can be presented as multi-stage process of emergence of the fractal unit.

At the first stage of eutectic crystallization in fusion primary phases are formed: inclusions of graphite and clusters of austenite. Graphite inclusions at crystallization perform function of primary fetus in aggregation model, and around them in the overcooled fusion eutectic cells form.

At the second stage, at the moment previous their aggregations, eutectic cells and primary clusters make Brownian motion in fusion. The direction of the movement of eutectic cells and clusters of austenite in fusion depends on total impact on them of different forces. In particular, the forming particles of a firm phase in fusion are affected by convective flows of fusion, gravitational and Archimedean forces. At mutual contact of eutectic cells with austenitic shells there is their welding. At the time of contact of separate particles and after it growth of eutectic cells on the normal diffusion mechanism continues. The chains of graphite inclusions observed at microstructural researches of cast iron represent the eutectic cells which connected among themselves arose at the initial stage of aggregation. As a result of association of chains of eutectic cells and austenitic clusters fractal cluster aggregates are formed.

The final stage of crystallization of cast iron - transition from the fractal unit to percolation or on terminology B. Mandelbrot's - succolarity [8]. Percolation - process of course of liquid in the firm environment. At the same time in pure form percolation represents through course that in the conditions of crystallization of casting is impossible. Therefore, the use of the term 
«succolarity», meaning «under-flowing», is the most acceptable. In relation to process of crystallization of cast iron at this stage there is an overflowing of the uterine fusion in intercluster space created later formations of the fractal aggregate. The uterine fusion enriched with likviruyushchy components of alloy moves in yet not hardened areas of a aggregate framework. At this stage in structure there can be cavities isolated from a liquid phase in which at the end of hardening a pores is formed. The most obviously cluster structure is shown in places of a shrinkable pores and sinks which at crystallization of high-strength cast iron are distributed unevenly in volume of casting.

The illustration of model of structure formation of fractal aggregate in high-strength cast iron, is presented in fig. 7. According to the developed model shown separate stages of forming of clusters (fig. 7 a), education fractal (fig. 7 b), and the succolarity aggregate (fig. 7 c).

The final illustration (fig. 7, d) presented by the image of a real microstructure contains a pores. This sample for production of a microsection was taken from the lower part of harvesting where formation of a pores of shrinkable origin is least probable. Emergence of such pores in the lower parts of casting is a consequence of uneven hardening of uterine fusion at formation of the succolarity aggregate.
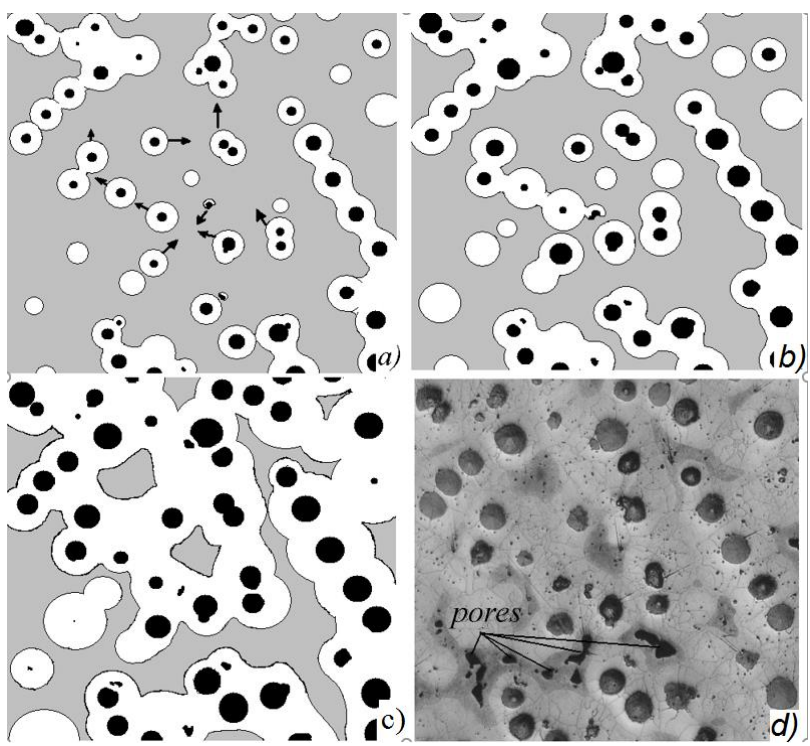

Fig. 7. Stages of crystallization of high-strength cast iron: $a$-emergence of clusters from eutectic cells and austenite; $b$ - fractal aggregation of primary clusters; $c$ - transition to succolarity cluster; $d$ - microstructure in a sample from highstrength cast iron (fragment fig. 3,a)

\section{Conclusion}

The technique of the fractal analysis of images of structure of materials is developed. Methodological aspects and restrictions of the developed way are defined. The offered technique is based on use of previously received computer models of structure of cast iron and application of programs for the analysis of images.

The model of processes of primary structurization of highstrength cast iron is developed. The fractal dimensions calculated during the research allow to present cast iron crystallization process as the aggregation of eutectic cells happening according to a cluster - cluster model of growth of the fractal unit. Process of structurization of high-strength cast iron is presented by several stages including forming of primary clusters, formation of eutectic cells, fractal aggregation of these educations and the subsequent transition from fractal to a percolation cluster.

\section{References}

[1] Bozhokin S. V. (2001) Fraktaly i multifraktaly [Fractals and multifractals]. Moscow-Izhevsk. NRC: Regular and chaotic dynamics (in Russian)

[2] Vstovskij G.V., Kolmakov A.G., Bynin I. Zh. (2001) Vvedenie $v$ mul'tifraktal'nuyu parametrizaciyu struktur materialov [Introduction to multifractal parameterization of materials structures]. Moscow-Izhevsk. NRC: Regular and chaotic dynamics (in Russian), p. 116.

[3] Falconer K. (1997) Techniques in Fractal Geometry, John Wiley \& Sons, $256 \mathrm{p}$.

[4] Feder E. (1991) Fraktaly [Fractals]. Moscow: World. 254 p.

[5] Gong Yu, Chengbao Wu, Jian Wang, Lei Kong. (2015) Calculating the Fractal Dimension of the Material Fracture Surface Based on the Triangular Prism Surface Area Method. International Conference on Automation, Mechanical Control and Computational Engineering (AMCCE 2015), pp. 2242 - 2247.

[6] Kulak M. I. (2002) Fraktalnaya mehanika materialov [Fractal mechanics of materials]. Minsk: High school. 302 p.

[7] Makarenko K.V. (2009) Simulation of Crystallization Process of Iron with Globular Graphite. Metal Science and Heat Treatment, V. 51, № 11-12. pp. 528 - 532.

[8] Mandelbrot B. (2002) Fraktalnaya geometriya prirody [The fractal geometry of nature]. Moscow: Institute for computer research, p. 656.

[9] Masatoshi Futakawa, Kihei Tsutsui, Hiroyuki Kogawa, Takashi Naoe (2016) Numerical Simulation on Molten Metal Collision Behavior Using SPH Method Combined with Fractal Analysis on Morphology of Stacking Pattern Key Engineering Materials, V. 715. pp. 203-209.

[10] Nakayama T., (2003) Fractal Concepts in Condensed Matter Phisics. Springer: Series in solid-state sciences, 224 p.

[11] Oliveira Alessandra da Silva, Verônica dos Santos Lopes, Ubirajara Coutinho Filho, Rodrigo Braga Moruzzi, André Luiz de Oliveira (2018) Neural network for fractal dimension evolution. Water Science \& Technology, № 4 (V. 78). pp. 795-802.

[12] Schaefer, Dale. W. (1988) Fractal Models and the Structure of Materials. Materials Research Society MRS Bulletin, №2 (V. XIII). pp. $22-27$.

[13] Smirnov B.M. (1991) Fizika fraktalnyh klasterov [Physics of fractal clusters]. Moscow: Science. $136 \mathrm{p}$.

[14] Swapna M. S., Sankararaman S. (2017) Fractal analysis - a surrogate technique for material characterization. Nanosystems: Physics, Chemistry, Mathematics, № 8 (6). pp. $809-815$.

[15] Wang Jian-zhong, Zheng-ping Xi, Hui-ping Tang, Weidong Huang, Ji-lei Zhu, Qing-bo Ao (2013) Fractal dimension for porous metal materials of $\mathrm{FeCrAl}$ fiber. Transactions of Nonferrous Metals Society of China, № 23. pp. $1046-1051$.

[16] Zolotyhin I.V., Kalinin U. E., Loginova V.I. (2005) Tverdotelnye fraktalnye stryktyry [Solid fractal structures]. Alternative energy and ecology, vol.29.no 9. pp. $56-66$. 